Biological evolution is an ongoing struggle for existence. The description of this struggle as a striving towards new records in fitness is helping us to understand the extinction statistics in the fossil record

Preben Alstrøm, Denmark

\section{Evolution - a Struggle for Records}

The physics literature on models of evolution has grown steadily in the last few years. Statistical data from fossil records have been considered in detail, and fundamental mechanisms underlying evolutionary events such as mass extinctions have been heavily discussed. The aim here is to point out a possible statistical similarity between evolution, the athletes' struggle to break records, the appearance of new innovations in technology and the acquisition of new skills. An underlying record dynamics seems to play an essential role in evolution, and seems to be responsible for many of the statistical characteristics observed in the fossil record.

Life on earth originated 3.5 billion years ago, but two billion years followed before complex multicelled organisms appeared in a catastrophic event 600 million years ago. This event, called the Cambrian explosion, marked the beginning of the Phanerozoic period, for which a remarkable fossil record has accumulated. As pointed out by several authors, the statistical material provided by the fossil record is rather limited when it comes to understanding the development of a given species at the level of organisms. However, at the species level, the fossil record turns out to be quite useful.

One characteristic of the fossil record is the presence of "quantal jumps" or "punctuations", where new species appear on a relatively short geological time scale. Evolution is not a slow process, where one species gradually changes into another. The evolutionary jumps have, in physics language, been associated with an energy picture as well as with an entropy picture. In the energy picture the species move through mutations from one maximum to another in a fitness landscape, where similar individuals appear as clusters. The landscape has strong barriers which the population has to cross, and this gives rise to long periods of stasis where species do not change. In the entropy picture the long periods of stasis are not due to barriers but are due to the fact that another fitness peak may be hard to find. In comparison with golf, the energy picture corresponds to getting the ball over a hill, while the entropy picture corresponds to getting the ball into the hole.

The period of stasis is sometimes short, sometimes very long. In the latter case the involved species, which include the shark, the cockroach, and the coelacanth fish, are known as "living fossils." The average lifetime for species from the fossil record is estimated to be about four million years, but some species have been around for more than a hundred million years. How do these living fossils come into being? To clarify this point it is helpful to consider the experiment conducted by R.E. Lenski and M. Travisano at Michigan State Univeristy, who studied the evolution of 12 populations of the bacterium Escherichia coli, which were all generated by identical (cloned) ancestors, and were allowed to grow in identical but physically separated environments. The fitness, which was defined as the Malthusian rate of population increase, was followed for 10000 generations. Figure 1 shows the time dependence of the average fitness in a single population of E. coli relative to its original ancestor. The data are given on a semi-logarithmic scale. The figure shows that the fitness only grows logarithmically. Roughly speaking, the time it takes for E. coli to evolve from one type into a more fit one is always comparable to the entire time elapsed from the beginning of the experiment. The presence of living fossils may very well be the result of such a logarithmic process.

What is the origin of the above logarithmical slow-down? One suggestion put forward by P. Sibani and coworkers at the University of Odense in Denmark is a simple mechanism where the dominant evolutionary search is not only for another fitness peak, but for a better fitness peak, ie for a new record in fitness. Because new records are harder and harder to find, species tend to stay put around the same peak for longer and longer. As- suming that the correlation 'length' in the fitness landscape is small compared to the distance to the next fitness peak (rugged fitness landscape), one can analytically show that the number of records grows logarithmically in time. Thus, the logarithmic behaviour revealed by the experiments of Lenski and Travisano can be explained simply by associating the achievement of a new record to a certain growth in fitness.

The logarithmic slow-down resulting from a struggle for records may be quite general. In the book Full House by S.J. Gould, the "right wall" reached by athletes when trying to improve their performance is discussed, the prime example being the batting skills in baseball. Seen in the light of the above, learning curves should be logarithmic in form. In the early phases of learning a new skill, for example playing chess or skiing, progess is rather fast. Later, the time invested (and needed) to become better grows, eventually reaching the time-scale of months or years, at which level no further improvement takes place. Similar arguments in the context of technological development have been forwarded by S. Kauffman in his book At Home in the Universe. After a fundamental innovation is made, for example the bicycle or the automobile, radical modifications are invented greatly improving the bicycle or the automobile. However, as better forms are found, it becomes harder and harder to find further improvements.

Can we observe the possible presence of an underlying record dynamics in the fossil record? The answer seems to be "yes", if we believe that speciation events are related to the achievement of new fitness records, as well as to extinction events, meaning that the extinction of one species makes room for another, or the appearance of a new species causes another species to go extinct, due to competition for limited resources, for example. Let us for illustrative purposes assume that the development of a new species is directly associated with the extinction of another. The number of extinctions (per species existing) would then be growing logarithmically, and the extinction rate would not be an evolutionary constantwhy should it? - but decline inverse proportionally with time. As a result, the percentage of species that went extinct in the beginning of the Phanerozoic period must have been larger than the percentage of species that has gone extinct within the last few million years. This decline in extinction rate has been pointed out by D.M. Raup and J.J. Sepkoski at the University of Chicago who considered the 


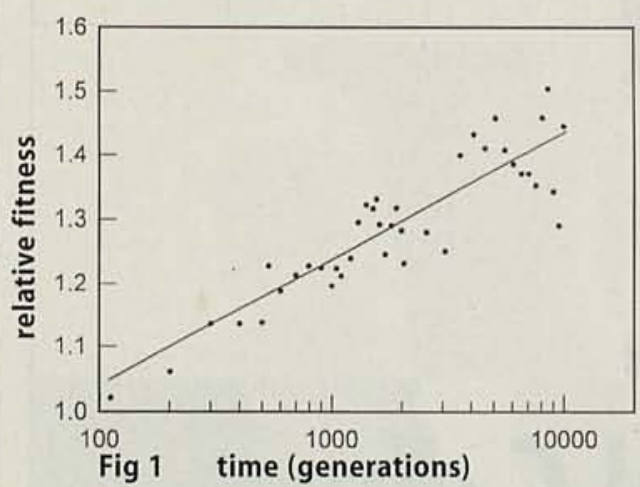

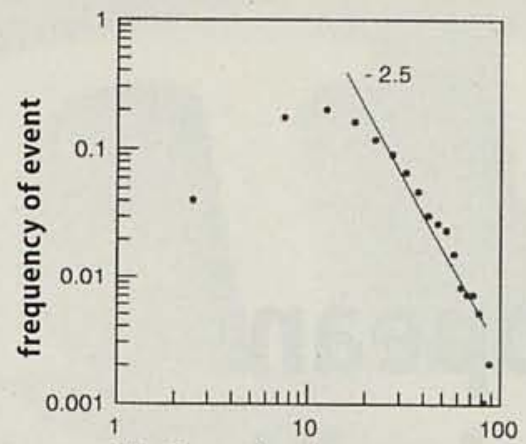

Fig 2 exctinction percentage

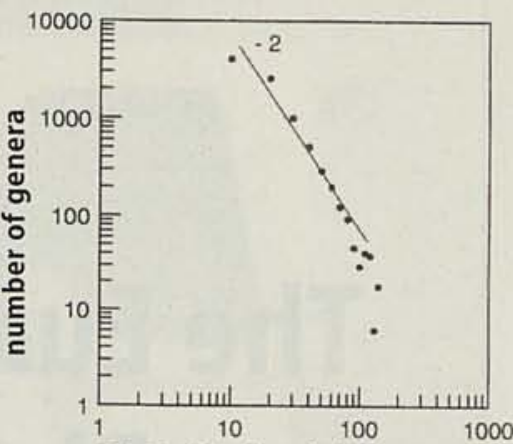

Fig 3 life time (million years)

Fig 1 The mean fitness relative to the fitness of original ancestors in an E. Coli population grown in a glucose-limited environment. A semi-logarithmic plot versus the number of generations is shown. Note that the data approximately follow a logarithmic fitness growth, suggesting an underlying struggle for records - Fig 2 \& 3 show data obtained from the fossil record - Fig 2 Frequency of extinction events versus extinction percent. The decay exponent -2.5 is the reciprocal of the decay exponent for the percentage extinction rate - Fig 3 The distribution of life spans of fossil genera of marine animals

SOURCEs: R.E. Lenski, M. Travisano Proc. Natl. Acad. SCi. USA 916808 (1994); Extinction: BAd Genes or BAd LuCK by D.M. Raup

change in extinction rate for families of marine vertebrates and invertibrates since the Cambrian time. The data are rather scattered, but a clear decrease in extinctions is observed. To highlight the decay, it is helpful to integrate the percentage extinction rate. Whether or not the five big mass extinctions are included in the integration doesn't matter, since only a few percent of the total number of extinctions is associated with these. As argued by P. Sibani, one can introduce abiotic catastrophic events such as meteor impacts or rises in the sea level. It does not influence the general decline in extinction rate.

The integrated percentage extinction rate does not grow linearly in time as would be the case if the extinction rate were stationary. It grows more slowly, roughly as a power law in time with exponent 0.6 , implying that the percentage extinction rate also decreases as a power law in time with exponent -0.4. In comparison, in the simple logarithmic picture above the exponent would be -1 . Obviously, some important ingredients are missing. One of these is the competition between species in a co-evolving environment. Competitive interactions influence the decay of the extinction rate. However, the fundamental observation that the extinction rate decreases also has direct consequences for the survival probabilities of species. This can be seen in a careful interpretation of statistical data as, for example, how often a given extinction percentage appears in the record (figure 2). The distribution found must not be considered to be time independent since, due to the decrease in extinction rate, larger events are on average associated with earlier times, and smaller events with later times. Neglecting for simplicity the fluctuations in extinction rate, the frequency of events can be shown to decay with the extinction percentage according to a power law with an exponent -2.5 (the reciprocal of the extinction rate exponent). The temporal distribution of large and small events must also be taken into account when discussing "kill curves," describing the average waiting time between two events with a given extinction percentage. Such waiting times are not constant, but seem to increase in evolutionary time.

The many statistical measures of evolution which have been studied are often interdependent and, with simple assumptions, the behaviour of one can sometimes be explained in terms of another. One example already given is the frequency of extinction events of a certain percentage (ie size), where the decay exponent turns out to be to be the recipro$\mathrm{cal}$ of the extinction rate decay exponent. Another example is the power spectrum obtained for the temporal fluctuations in the number of species or families. Here M. Newman at Santa Fe Institute has provided simple arguments, relating the observed $1 / f$ type power-law decay of the spectrum with the power law found for the percentage extinction rate: the spectrum exponent is -2 times the exponent for the integrated percentage extinction rate. If the latter is 0.6 , the spectrum exponent comes out as -1.2.

Finally, we comment on the commonly studied life-time distribution of species. Figure 3 is based on the life spans of 17505 extinct genera of marine animals compiled by J.J. Sepkoski and presented by
D.M. Raup in his book Extinction: Bad Genes or Bad Luck. Much discussion on evolution in the physics literature has been concerned with this distribution, and the exponent -2 which seems to be characteristic of the distribution. Based on the record-driven dynamics introduced by P. Sibani and coworkers and discussed above, an additional introduction of a co-evolutionary interactions is enough to produce a life-time distribution with a characteristic exponent of -2 , which is surprisingly robust to changes in the interaction range.

It seems that the paradigm of a struggle for new records is worth further consideration. Ample statistical material sug-* gests that biological evolution has not reached a stationary state. The extinction rate seems to decrease due to the prolonged survival of species through the Phanerozoic period. And the presence of an underlying record dynamics can be identified in the statistical behaviour of the fossil record.

The author is chairman of the new EPS Division in Statistical and Nonlinear Physics and also the Danish correspondent for Europhysics News. He is an associate professor at the Niels Bohr Institute in Copenhagen

\section{Further reading}

Evolution and Extinction Dynamics in Rugged Fitness Landscapes by P. Sibani, M. Brandt, P. Alstrøm Int. J. Mod. Phys. 12 361-391 (1998) - Full House by S.J. Gould (Harmony Books, New York, 1996) - At Home in the Universe by S. Kaufman (Oxford University Press, 1995) - Fitness Optimization and Decay of Extinction Rate Through Biological Evolution by P. Sibani, M.R. Schmidt, P. Alstrøm Phys. Rev. Lett. 752055 (1995) 